Itinéraires Itinéraires

Littérature, textes, cultures

" Much Ado about Nothing »? Le cas de l'Espagne dans l'historiographie de la critique des hypotextes shakespeariens

\title{
Anna Demoux
}

\section{CpenEdition}

Journals

Édition électronique

URL : http://journals.openedition.org/itineraires/2426

DOI : $10.4000 /$ itineraires.2426

ISSN : 2427-920X

Éditeur

Pléiade

\section{Référence électronique}

Anna Demoux, « « Much Ado about Nothing »? Le cas de l'Espagne dans l'historiographie de la critique des hypotextes shakespeariens », Itinéraires [En ligne], 2014-2 | 2015, mis en ligne le 17 juillet 2015, consulté le 02 octobre 2020. URL : http://journals.openedition.org/itineraires/2426 ; DOI : https://doi.org/10.4000/itineraires.2426

Ce document a été généré automatiquement le 2 octobre 2020.

\section{cc) $(1) \&$}

Itinéraires est mis à disposition selon les termes de la licence Creative Commons Attribution - Pas d'Utilisation Commerciale - Pas de Modification 4.0 International. 


\title{
« Much Ado about Nothing »? Le cas de l'Espagne dans l'historiographie de la critique des hypotextes shakespeariens
}

\author{
Anna Demoux
}

\section{NOTE DE L'AUTEUR}

Henry Thomas pensait que l'influence directe de l'Espagne sur William Shakespeare était mineure et que le titre Beaucoup de bruit pour rien conviendrait mieux que Shakespeare and Spain pour son ouvrage : « a more fitting title would have been one which Shakespeare himself has provided ready to hand-Much Ado about Nothing-for I am on the side of those who think that Spain's direct influence in Shakespeare is small » (Thomas $1922: 3$ ).

«Rien ne se perd, rien ne se crée, tout se transforme ${ }^{1}$.»

Dans les années 1950 et 1960, l'intérêt pour les hypotextes² littéraires et dramatiques shakespeariens - qui portent à cette époque le nom de «sources ${ }^{3}$ »- est à son paroxysme: Geoffrey Bullough publie Narrative and Dramatic Sources of Shakespeare, un ouvrage de référence en huit volumes ayant pour but de répertorier et mettre à disposition de tous les textes littéraires et historiques ayant influencé le corpus théâtral de William Shakespeare ou présentant des intrigues et des personnages analogues. Celui-ci inclut trente-sept pièces ${ }^{4}$. Sur un total de 164 œuvres éditées, dans leur intégralité ou en partie, quatre seulement ${ }^{5}$ sont des œuvres à l'origine en langue espagnole, et une seule est répertoriée en tant que "source »-Diana de Jorge de Montemayor $^{6}$ : lorsque l'influence de la littérature espagnole chez Shakespeare est 
envisagée selon la perspective de ce que Gérard Genette appelle les relations intertextuelles ${ }^{7}$, elle est considérée comme quasi inexistante.

2 Ce dernier opère et explicite un changement de perspective dès le chapitre I de Palimpsestes par rapport à ses propres travaux mais aussi ceux de ses collègues : il redéfinit notamment la notion d'intertextualité telle qu'elle a été théorisée par Julia Kristeva dans Sémiotikè. Il inclut de nouveaux supports et outils en opérant le passage de l'intertextualité traditionnelle à la transtextualité8. La nature de la «source» n'est plus restreinte à l'idée de texte écrit - s'agit-il encore d'une "source ", d'ailleurs ? La notion est étendue à celle d'hypotexte. Il est ainsi possible de reconsidérer la présence de l'Espagne dans l'écriture shakespearienne en élargissant le champ des possibles des hypotextes. Il s'agira de constituer une typologie analytique de ces outils opératoires permettant de penser d'un côté l'influence de la littérature espagnole sur le corpus théâtral shakespearien et de l'autre l'interculturalité entre l'Espagne du Siècle d'or et l'Angleterre élisabéthaine et jacobéenne, mais ici dans un sens unique, de l'Espagne vers l'Angleterre, dans ses différentes manifestations émergeant de ce corpus.

3 Si l'exhaustivité est impossible dans cette étude, les ouvrages choisis sont représentatifs: ce sont soit des ouvrages consacrés dans leur intégralité à cette problématique soit des ouvrages qui réactualisent la réflexion par une nouvelle approche mais qui ne sont pas exclusivement réservés à l'étude du corpus théâtral shakespearien. Deux courts ouvrages intégraux adoptant la méthode comparatiste traditionnelle sont publiés sur le sujet avant la parution des huit volumes de Geoffrey Bullough, qu'il est possible de classer dans la même catégorie méthodologique : William Shakespeare and Alleged Spanish Prototypes d'Albert R. Frey paru en 1886 et Shakespeare and Spain de Henry Thomas paru en 1922. Leur rayonnement s'avère considérable puisqu'il faut attendre 1991 pour qu'un troisième ouvrage remettant en cause leurs conclusions, ainsi que leur méthodologie, paraisse en Espagne. Il s'agit de España en Shakespeare de Pedro J. Duque, qui, sur le plan méthodologique, représente la transition de l'intertextualité vers la transtextualité. Par ailleurs, deux démarches se distinguent : la première, influencée par Drama of a Nation de Walter Cohen paru en 1985, et dans laquelle s'inscrit également Public Theatre in Golden Age Madrid and Tudor-Stuart London: Class, Gender and Festive Community d'Ivan Cañadas paru en 2008, étudie l'histoire des théâtres anglais et espagnol de la Renaissance en parallèle selon une approche historique marxiste. La seconde s'intéresse à la présence de l'Espagne sous sa forme "transtextuelle en creux", autrement dit, implicite, et ce grâce aux apports de l'anthropologie symbolique telle qu'elle a été définie par Clifford Geertz. Dans English Renaissance Drama and the Specter of Spain, paru en 2008, Eric J. Griffin développe un concept clé, celui d'« ethno-poétique », et cite l'Anatomie de la critique de Northop Frye et Métahistoire de Hayden White.

\section{À la recherche des sources : la perspective intertextuelle}

\section{«Petit croquis d'une controverse oubliée »}

4 En 1998, Hugh Wilson retrace l'historiographie de la critique des hypotextes shakespeariens espagnols au début de son article «Shakespeare's Taming of the Shrew and Traces of Spanish Influence » et il intitule cette partie de son travail : « Thumbnail 
Sketch of a Forgotten Controversy». Frey et Thomas sont les deux premiers universitaires à consacrer un court ouvrage intégral au débat autour de la réalité de la coprésence entre la littérature espagnole et l'œuvre théâtrale de Shakespeare. Ils s'attellent tous deux à la réfutation systématique des travaux antérieurs démontrant la présence explicite de personnages, d'épisodes ou d'intrigues tirés de la littérature espagnole. En amont et pendant la période des années 1880 aux années 1920, d'autres études abordent cette question mais ponctuellement ou sous la forme d'un chapitre: c'est le cas de Spanish Literature in the England of the Tudors de John Garrett Underhill (1899), Spanish Influence on English Literature de Martin Hume (1904), The Literature of Roguery, de Frank Wadleigh Chandler (1907), The Relations between Spanish and English Literature de James Fitzmaurice-Kelly (1910) et enfin Spanien und das Elisabethanische Drama de Rudolf Grossman (1920). Joseph de Perott, quant à lui, y consacre également un nombre important d'articles ${ }^{9}$. Précédemment, en Allemagne, au xIX siècle, M. Karl Simrock affirme que Shakespeare a utilisé Diana de Jorge de Montemayor pour The Two Gentlemen of Verona, Julius Leopold Klein démontre qu'il a lu la pièce de Lope de Vega Castelvines y Monteses avant d'écrire Romeo and Juliet et Edmund Dorer propose Las Noches de invierno de Antonio Eslava comme source de The Tempest.

\section{Présentation des méthodologies de Thomas et Frey}

Selon Thomas, "l'étendue de la familiarité qu'un auteur a de la langue d'un pays étranger est évidemment un facteur important lorsque l'on considère les influences possibles exercées sur lui par la littérature de ce pays ${ }^{10}$ » (Thomas 1922: 4). De ce postulat de départ découle une première catégorie primaire d'outils d'analyse textuelle, les preuves linguistiques, "linguistic evidence» (Thomas 1922:5), qui illustrent la présence explicite de la langue espagnole dans le corpus shakespearien. Il y classe le vocabulaire d'origine espagnole, dont il ne cite aucun exemple, car il ne relève aucune occurrence dans le corpus, les noms d'objets d'origine espagnole (comme les épées, le vin, les oranges, etc.), les références géographiques à l'Espagne et les personnages espagnols. Il en arrive à la conclusion qu'en l'absence de preuves linguistiques en quantité conséquente, affirmer que la littérature espagnole ait une influence déterminante sur Shakespeare est infondé : « La connaissance que Shakespeare avait de l'Espagne était, semblerait-il, celle du citoyen londonien éclairé ${ }^{11} »$ (Thomas $1922: 10$ ). Il en va déjà de même dans l'ouvrage de Frey paru plus de trente ans auparavant. Celuici affirme : «les mots et expressions espagnols dans les pièces de Shakespeare peuvent être comptés sur les doigts d'une main, et ils sont soit des corruptions, soit, (dans deux exemples) des caricatures de célébrités espagnoles ${ }^{12} »$ (Frey $1886: 16$ ).

Devant les conclusions négatives apportées par les preuves linguistiques, Thomas propose une deuxième catégorie primaire d'outils d'analyse textuelle, les emprunts littéraires, "Shakespeare's literary borrowings from the Peninsula» (Thomas 1922: 10), qui appartiennent à la catégorie de la coprésence textuelle ${ }^{13}$. Il cite le nom de quatorze ouvrages espagnols ${ }^{14}$, en les classant en deux sous-catégories. La première, celle des cas mineurs, "minor cases» (Thomas 1922: 15), répertorie des titres qui présentent peu d'intérêt à ses yeux excepté le cas du Don Quichotte pour Cardenio, le cas le plus intéressant de tous, "the most interesting of all» (Thomas 1922: 20). La deuxième est constituée de deux genres littéraires, la pastorale et les romans de chevalerie, qui ont plus de crédit aux yeux de Thomas. Ces emprunts littéraires sont traités tantôt à l'échelle de l'intrigue (Diana et The Two Gentlemen of Verona, Don Quijote et 
Cardenio), de l'épisode (dans Merry Wives of Windsor, III, 3-5, Falstaff se cachant dans un tonneau chez Mistress Ford rappelle un passage similaire de la Segunda Celestina de Feliciano de Silva) ou de la réplique (l'expression « Ho! now you strike like the blind man: 'twas the boy that stole your meat, and you'll beat the post » dans Much Ado About Nothing II, 1, 187-188, est inspiré par l'épisode de l'aveugle dans le chapitre I du Lazarillo de Tormes), tantôt sur le plan de l'onomastique, de la caractérisation, de la thématique, du lexique et du style. Frey utilise les mêmes outils mais sur un corpus plus restreint car sa problématique de départ se concentre sur un auteur espagnol en particulier, Lope de Vega ${ }^{15}$ :

[...] les mêmes personnages, intrigues, et lieux ont été utilisés par ces hommes dans la construction de certaines de leurs productions dramatiques, et, comme ils étaient contemporains, cela donne l'impression qu'ils se sont adonnés à une quantité considérable d'emprunts - appelez cela du plagiat si vous le souhaitez. [...] Comme Shakespeare a plus ou moins été accusé de non-originalité, il serait important de considérer dans quelle mesure, si c'était le cas, il est redevable envers Lope de Vega et son école ${ }^{16}$. (Frey $1886: 5-6$ )

\section{Analyse de ces méthodologies}

7 Alors qu'un nombre ${ }^{17}$ important de pièces shakespeariennes est évoqué et que la controverse traverse l'ensemble de l'œuvre théâtrale de Shakespeare de The Two Gentlemen of Verona à Cardenio, qu'il s'agisse des pièces historiques, des comédies ou des tragédies, l'étude des ouvrages de Frey et Thomas montre qu'ils s'appuient sur une méthodologie qui permet d'identifier ce que Gérard Genette appelle une «transformation » textuelle (Genette $1982: 14$ ) ou une « dérivation » textuelle (Genette 1982 : 19). En effet, les outils utilisés par Frey et Thomas permettent de mettre en évidence des références directes et explicites. C'est d'ailleurs ce qu'annonce Thomas dès le premier paragraphe de son ouvrage (voir la note de l'auteur de cet article). Coriolanus et les autres pièces romaines inspirées des Vies parallèles de Plutarque dans leur traduction de Thomas North, Julius Caesar, Anthony and Cleopatra, constituent un corpus sur lequel cette méthodologie traditionnelle fonctionne très bien puisque ces traductions ont été identifiées comme support hypotextuel privilégié. Ainsi, que ce soit à l'échelle de l'intrigue, de l'épisode, de la réplique ou même du vers, l'hypertexte théâtral est très proche de l'hypotexte biographique. La Renaissance étant la période historique de la redécouverte des classiques, sur le plan éthique et esthétique, l'adaptation de ces textes au théâtre possède une légitimité que ne possède pas l'adaptation de la littérature espagnole. Elle peut donc être "directe » et "explicite " sans poser de problème de censure. Mais ce n'est pas le cas des hypotextes espagnols : Thomas et Frey amalgament ainsi l'absence d'influence directe avec l'absence d'influence tout court et concluent à la non-existence de liens intertextuels entre la littérature espagnole et Shakespeare en s'appuyant sur des données quantitatives. S'il y a peu de références à l'Espagne et à la littérature espagnole, c'est qu'il n'y a pas d'intertextualité identifiable ou qu'elle est vraiment minime.

8 La méthodologie et les outils employés dans les deux études sont similaires, mais néanmoins pas complètement identiques. Thomas prend soin de commenter chacun des exemples tirés des travaux de ses prédécesseurs afin de les recontextualiser dans le milieu dans lequel évolue Shakespeare : la ville de Londres et les marchés de la région de Stratford-upon-Avon, sa ville natale. Même si les limites socioculturelles ne sont pas aussi clairement définies que le cadre géographique, il insiste avant tout sur l'anglicité 
du dramaturge car il considère que tout contact avec la culture espagnole s'est fait sur le territoire anglais. Selon lui, l'argument de l'influence espagnole est irrecevable à partir du moment où elle se déroule sur le territoire anglais ou à travers la langue anglaise, comme c'est le cas pour les traductions de textes espagnols en anglais. Frey, de son côté, se fait l'avocat d'un Shakespeare accusé de "non-originalité » et de "plagiat", dont il souhaite avant tout défendre le statut de génie littéraire et dramatique. S'il reconnaît volontiers la possibilité d'une influence étrangère sur le dramaturge, celle-ci doit avant tout être italienne ou classique car le théâtre espagnol est selon lui « défectueux» (Frey 1886: 40, je traduis). Il conclut ainsi : «trouverait-il alors nécessaire de copier des formes aussi peu fidèles? Tirerait-il un quelconque bénéfice en imitant des prototypes aussi irréalistes ? Assurément non !18 (Frey 1886 : 41). Il apparait ici indispensable de resituer ces discours et ces conclusions analytiques dans leur cadre d'origine, à la fois historique, socioculturel et méthodologique, d'autant plus que leur impact a perduré, comme l'ont signalé Pedro J. Duque et Hugh Wilson. Thomas, "outre le fait qu'il n'aborde pas plus que quelques-uns des aspects, est trop compendieux et superficiel, et, au moment où il termine sa lecture, donne l'impression d'être quelque peu dogmatique et conservateur ${ }^{19}$ ", affirme le premier (Duque 1991: 15). «Les opinions de Frey ont prévalu et découragé la recherche ${ }^{20}$ » selon le deuxième (Wilson 1998 : 235).

\section{Recontextualisation et exégèse des théories de Frey et Thomas}

\section{Une méthodologie au service de l'idéologie}

Hugh Wilson accuse Thomas de tenir des propos à connotation nationaliste, « chauvinist overtones" (Wilson 1998 : 236). En effet, celui-ci développe l'ensemble de ses contre-arguments autour d'un seul et même concept clé : l'anglicité de Shakespeare. Comme le rappelle Françoise Lavocat:

À l'origine, en effet, et jusqu'à la deuxième guerre mondiale, la critique a visé, souvent de façon violente, l'idéologie cosmopolite supposée inhérente à la discipline. Il ne faut en effet pas minimiser le chauvinisme qui inspire, jusqu'à aujourd'hui, le rejet ou le dédain de nombreuses communautés académiques pour le comparatisme. Dans bien des pays, ces communautés sont encore et en grande part mobilisées par la construction ou la préservation d'un canon national. (Lavocat 2012)

10 Thomas, qui écrit juste après la Première Guerre mondiale, met sa méthode au service de l'idéologie et ses outils sont biaisés dans l'intérêt de la construction de l'identité nationale. Cette argumentation possède pourtant en germe toutes les pistes nécessaires à la reconsidération du cas de l'Espagne dans l'œuvre shakespearienne. Il suffit de reprendre les arguments de Thomas les uns après les autres pour voir naître de nouvelles pistes d'étude: il évoque par exemple l'influence des personnalités espagnoles présentes dans la capitale londonienne comme Rodrigo Lopez ou Antonio Lopez sur le «type » de l'Espagnol euphuiste, fanfaron et couard dont la figure la plus marquante est le personnage de Don Adriano de Armado dans Love's Labour's Lost.

11 Frey, de son côté, met en place une hiérarchie entre les différentes origines géographiques des hypotextes. Les hypotextes classiques et italiens ont droit de cité mais les hypotextes espagnols sont mis à l'index ou bien relégués au rang d'analogues 
car ils présentent de nombreux défauts stylistiques: «les unités de temps et de lieu n'étaient pas respectées, et les faits historiques étaient pervertis pour les besoins de l'adaptation. Mais ces anachronismes et ces bourdes sont très peu présentes dans les œuvres de Shakespeare ${ }^{21} »($ Frey 1886: 40). Quiconque a pris le temps de comparer les hypertextes shakespeariens et leurs hypotextes, quels qu'ils soient, sait que cette affirmation est absolument erronée: il n'y a aucune règle des trois unités dans le théâtre de Shakespeare et les faits historiques sont bien " pervertis (je reprends le terme ci-dessus mais sans connotation de jugement moral) pour les besoins de l'adaptation » (Ibid.).

\section{Traduction, imitatio et censure}

12 Si l'origine «étrangère » de ces hypotextes est au cœur de la problématique, elle a des conséquences notables, aussi bien à l'époque de Shakespeare qu'à l'époque des critiques, sur leur "statut » littéraire. Hugh Wilson rappelle que dans l'Angleterre protestante il était peu aisé de se réclamer ouvertement influencé par les textes de l'Espagne catholique : «il est possible que les auteurs protestants censurés aient hésité à citer des autorités espagnoles suspectes ${ }^{22}$ "(Wilson 1998: 235). En effet, il était d'usage, aussi bien pendant la période d'apprentissage que lorsqu'on devenait un auteur accompli et reconnu, d'écrire en «imitant ", en écrivant "à la manière de » ses prédécesseurs illustres selon la doctrine de l'imitatio. Ce terme est d'ailleurs repris par Gérard Genette dans Palimpsestes pour désigner une forme spécifique d'hypertextualité : il s'agit d'une «transformation plus complexe et plus indirecte » qui « raconte une tout autre histoire [...] mais en s'inspirant pour le faire du type (générique, c'est-à-dire à la fois formel et thématique) établi par [l'auteur de l'hypotexte] dans [celui-ci], ou, comme on l'a bien dit pendant des siècles (je souligne) en [l']imitant (Genette souligne) " (Genette 1982 : 14). Il prend l'exemple du lien hypertextuel entre l'Odyssée d'Homère et l'Énéide de Virgile pour illustrer cette définition. Ainsi, si imitation des classiques espagnols il y avait, la censure demandait à ce que cela soit fait de manière très discrète et implicite. C'est la raison pour laquelle les outils méthodologiques proposés par Frey et Thomas ne sont pas adaptés à l'étude des hypotextes espagnols chez Shakespeare. Il est tout simplement impossible de faire émerger des références implicites avec des outils qui relèvent de l'explicite.

L'utilisation d'hypotextes espagnols traduits en anglais, telle qu'elle est décrite dans les travaux de Frey et Thomas, remet en question la notion même de traduction. Si Frey reconnaît à la traduction des œuvres en anglais leur origine étrangère, Thomas y voit une véritable appropriation nationale : le passage à la langue anglaise dénature le texte original et le prive de son identité culturelle. Dans le cas de Romeo and Juliet, le professeur Fitzmaurice-Kelly a proposé la Comedia de Calisto y Melibea comme hypotexte possible parmi d'autres. Thomas affirme : « [Shakespeare] avait de nombreuses sources d'inspiration pour Roméo et Juliette parmi ses prédécesseurs dans le même domaine, Brooke et Painter ${ }^{23}$ » (Thomas 1922 : 16). Or, The Palace of Pleasure de William Painter est un recueil de nouvelles empruntées à des auteurs italiens et classiques. Certes, ils ne sont pas espagnols, mais ils ne sont pas anglais non plus. Ainsi, la traduction en langue anglaise semblerait relever d'une véritable assimilation, d'une forme d'« anglicisation ", sans possibilité d'identifier l'origine étrangère de l'ouvrage. Frey reprend les travaux du professseur Klein et les réfute en proposant l'idée d'une influence indirecte des œuvres italiennes sur Shakespeare. En effet, il évoque Storia di 
Verona de della Corte, La Giulietta de da Porto et les Novelle de Bandello comme hypotextes du Palace of Pleasure de Painter. Il évoque aussi la traduction anglaise de Bandello par Arthur Brooke. Les analogies entre l'intrigue de la pièce de Shakespeare et la pièce Castelvines y Monteses de Lope de Vega n'existent donc pas car c'est en fait un analogue de textes italiens, qui constituent d'après Frey les hypotextes premiers, indirects néanmoins, de Shakespeare. Ces nombreuses analogies entre les littératures espagnole et italienne ont selon lui des explications historiques en rapport avec l'expansionnisme de l'Espagne du Xvi ${ }^{e}$ siècle. Celle-ci avait des velléités sur le territoire italien et le rayonnement de la littérature italienne aurait profondément modifié et influencé la littérature espagnole. Ces interrelations complexes semblent ici expliquer en partie la raison pour laquelle il est très souvent possible d'y trouver des analogues. Par ailleurs, il existe en général des traductions en langue anglaise. Il est clair que les outils permettant de réexaminer la réalité de la coprésence entre les textes espagnols et les pièces de Shakespeare doivent être repensés. Certains universitaires ont déjà formulé des propositions.

\section{De nouvelles perspectives}

\section{De l'intertextualité à la transtextualité : España en Shakespeare}

Pedro J. Duque reproche à Frey et Thomas leur manque d'exhaustivité. Il s'appuie ainsi sur l'ensemble des œuvres de Shakespeare, de manière systématique. Le corpus est classé par catégorie générique : comédies, pièces historiques, tragédies et poèmes. Pour chaque œuvre, il cite les différents hypotextes proposés par ses prédécesseurs et les réétudie à la lumière des nouvelles découvertes scientifiques comme la datation des pièces, de nouveaux manuscrits et des traductions.

Par ailleurs, il intègre également tout type de connexion entre l'Angleterre et l'Espagne comme élément potentiellement hypotextuel, et ce, en englobant toute forme possible :

[...] toute prise de contact avec des personnages hispaniques ou hispanisants, ou avec leur projection dans ses œuvres, idées et manifestations d'un quelconque caractère, quel qu'en soit l'emploi ou l'allusion fait, toute attitude prise, et toute mention - explicite ou tacite - faisant référence à l'Espagne, aux Espagnols, à l'espagnol ou au castillan, seront ici pris en compte. Les influences directes ou indirectes sont seulement un autre mode d'être présent, mais pas le seul ${ }^{24}$.

16 Ainsi, cet ouvrage marque le passage vers un élargissement de la notion d'hypotexte espagnol chez Shakespeare sur le plan méthodologique et réhabilite le cas de l'Espagne dans le corpus shakespearien. Il définit son ouvrage comme un point de départ et non pas comme un point final à la recherche dans ce champ disciplinaire.

\section{L'approche historique marxiste de Cohen et Cañadas}

Selon Walter Cohen, ce qui unit les théâtres anglais et espagnol à la Renaissance, ce ne sont pas des liens d'hypotextes/hypertextes mais des développements analogiques parallèles. Ils peuvent être qualifiés de «théatres nationaux » qui touchent un large public, notamment populaire, quand partout en Europe, la Renaissance établit des formes artistiques à partir de la synthèse de la tradition populaire et de la culture érudite néoclassique. C'est le contexte politique de l'absolutisme, absent ailleurs en Europe, qui serait à l'origine de ce phénomène: ce régime a tout d'abord promu le 
théâtre " public » puis l'a beaucoup critiqué. La polysémie, la richesse et la complexité de ces textes soumis à la censure naissent ainsi de la dialectique entre texte et contexte. Les limites de cette théorie résident toutefois dans leur comparaison avec le développement du théâtre public français qui, bien que né sous le même type de régime, ne connaît pas vraiment une trajectoire similaire. Walter Cohen en arrive à identifier et interroger certaines conditions sociales qui auraient permis le développement de genres spécifiques à des classes sociales dont la signification et l'impact ne leur sont pas nécessairement exclusifs - les comédies romantiques et les pièces historiques «nationales » à la fin $\mathrm{du} \mathrm{XVI}^{\mathrm{e}}$ siècle par exemple. Si l'étude en miroir des théâtres anglais et espagnol de la Renaissance semble de prime abord remettre en question la possibilité de liens hypertextuels vectoriels de l'espagnol vers l'anglais, il n'en est rien. En effet, le développement du genre dans les deux nations montre des fonctionnements similaires par "imitation» de prédécesseurs identiques. Cette approche a le mérite de permettre de distinguer, avec un degré de probabilité fort, les analogues des hypotextes.

\section{L'« ethno-poétique » d'Eric J. Griffin}

18 L'ouvrage de Griffin examine la construction complexe de l'identité raciale et culturelle de l'Espagne au début de l'époque moderne du point de vue des Anglais: il prend comme point focal la représentation que l'Angleterre a de l'Espagne. Cette dernière est à la fois l'ennemi juré qui l'empêche d'accomplir sa volonté impérialiste et son opposé religieux, tout du moins, telle est la représentation archétypale des stratégies politiques complexes entre les deux nations, bien moins dichotomiques en réalité. Selon lui, l'Angleterre a néanmoins le souvenir quelque peu nostalgique d'une histoire commune marquée par les alliances familiales et les mariages, qui reprendront avec les négociations supposées donner lieu au mariage du Prince Charles, fils de Jacques $\mathrm{I}^{\mathrm{er}}$, avec l'Infante d'Espagne Maria Anna, fille de Philippe III, union communément surnommée "the Spanish Match». Cette relation est décrite par Griffin comme une tension entre hispanophilie et hispanophobie dont la pierre d'achoppement est le combat contre l'Armada en 1588. Son corpus théatral est composé de The Spanish Tragedy de Thomas Kyd, The Jew of Malta de Christopher Marlowe, The Merchant of Venice et Othello de Shakespeare. Sa problématique repose sur un concept complexe défini comme suit :

[...] l'éthos de la cruauté espagnole pendant l'Inquisition et la conquête fut associé à la «noirceur » espagnole, l'ethnos de ses origines les «plus bâtardes » car c'est une nation composée non pas d'une seule race mais d'un mélange de romain, d'africain, de maure et de Juif. Cette confluence de discours produit l'« ethnopoétique» de l'impérialisme espagnol «envieux, amoral et impraticable ", par opposition avec «l'état souverain» idéalisé de l'Angleterre, "moins ambitieux sur le plan international et (sans doute) moins corruptible ${ }^{25}$. (Valbuena $2011: 182$ )

Bien que ce concept permette une véritable relecture du corpus avec un nouvel éclairage, Griffin suit une démarche qui s'inspire à la fois de l'historien et du sociologue. Par conséquent, ses analyses manquent d'outils stylistiques et rhétoriques. Il s'appuie uniquement sur l'onomastique et la polysémie des noms des personnages qui créent une sorte de réseau sémantique implicite faisant systématiquement référence à l'Espagne. 


\section{Conclusion} scientifiques sont unanimes. Les rapports entre les littératures et les cultures (au sens le plus large possible du terme) espagnoles et anglaises constituent un champ peu exploré alors qu'il nécessite une vraie attention :

Les hispanistes et les anglicistes qui travaillent dans les deux domaines des études modernes attendent avec impatience de nouveaux apports dans le champ relativement peu exploré de la littérature comparée anglo-hispanique ${ }^{26}$. (Valbuena $2008: 303)$

L'etude de l'évolution des outils théoriques opératoires utilisés pour déterminer la réalité de l'hypertextualité de l'Espagne sur le corpus théâtral shakespearien démontre que le changement de perspective et la réinvention de nouveaux outils, ou plutôt l'élargissement de la notion de "source " vers la notion d'hypotexte, a permis de relancer le débat universitaire de manière beaucoup plus dynamique et d'ouvrir le champ des possibles. Si influence de l'Espagne il y a, elle ne peut être ostentatoirement explicite et directe à cause de la complexité des liens géopolitiques, économiques et culturels entre les deux nations depuis le Moyen Âge jusqu'au milieu du Xvil ${ }^{\mathrm{e}}$ siècle. C'est la raison pour laquelle la prise en compte des dimensions socioculturelles et des représentations du pays en Angleterre est fondamentale. On pourrait s'étonner de voir qu'il n'y a pas plus de travaux sur le sujet en Espagne :

Alors que des travaux isolés sont apparus depuis les années 1980, la nécessité d'un travail plus important se fait sentir pour situer, historiciser, et reconnaître le potentiel dialogique des deux littératures ensemble $e^{27}$. (Valbuena $2008: 303$ )

\section{BIBLIOGRAPHIE}

Bullough, Geoffrey, 1957-1964, Narrative and Dramatic Sources of Shakespeare, 8 vol., Londres, Routledge and Kegan Paul, New York, Columbia University Press.

Cañadas, Ivan, 2005, Public Theater in Golden age Madrid and Tudor-Stuart London: Class, Gender and Festive Community, Burlington, VT, Ashgate.

Cohen, Walter, 1985, Drama of a Nation: Public Theater in Renaissance England and Spain, New York, Ithaca, Londres, Cornell University Press.

Duque, Pedro J., 1991, España en Shakespeare, Bilbao, Universidad de León - Universidad de Deusto.

Frey, Albert R., 1886, William Shakespeare and Alleged Spanish Prototypes, New York, Press of the New York Shakespeare Society.

Genette, Gérard, 1982, Palimpsestes, La littérature au second degré, Paris, Seuil, coll. « Points Essais ».

Griffin, Eric J., 2009, English Renaissance drama and the Specter of Spain: Ethnopoetics and Empire, Philadelphie, University of Pennsylvania Press.

Kristeva, Julia, 1969, Sémiotikè, recherches pour une sémanalyse, Paris, Seuil. 
Lavocat, Françoise, 2012, « Le comparatisme comme herméneutique de la défamiliarisation », Vox-poetica, [En ligne], http://www.vox-poetica.org/t/articles/lavocat2012.html.

Shakespeare, William, [1986] 2005, Complete Works, éd. Stanley Wells et Gary Taylor, Oxford, clarendon Press.

Thomas, Henry, 1922, Shakespeare and Spain, Oxford, Clarendon Press, coll. « The Taylorian Lecture».

Valbuena, Olga L., 2008, « Review of Public Theater in Golden age Madrid and Tudor-Stuart London », Medieval and Renaissance Drama in England, $\mathrm{n}^{\circ}$ 21, p. 301-303.

-, 2011, « Review of English Renaissance Drama and the Specter of Spain: Ethnopoetics and Empire ", Medieval and Renaissance Drama in England, $\mathrm{n}^{\circ} 24$, p. 181-184.

Wilson, Hugh, 1998, « Shakespeare's The Taming of the Shrew and Traces of Spanish Influence: Or, Exemplary Tales and Picaresque Fictions », SEDERI, $\mathrm{n}^{\circ}$ 9, p. 233-255.

\section{NOTES}

1. Maxime de Lavoisier inspirée du philosophe grec Anaxagore.

2. Dans Palimpsestes, Gérard Genette entend par « hypotextes » des textes à partir desquels sont dérivés d'autres textes, qu'il appelle les «hypertextes ». L'hypertextualité est, selon lui, " toute relation unissant un texte $\mathrm{B}$ (que j'appellerai hypertexte) à un texte antérieur A (que j'appellerai, bien sûr, hypotexte) sur lequel il se greffe d'une manière qui n'est pas celle du commentaire » (Genette $1982: 13$ ).

3. «[E]nsemble des œuvres, textuelles ou scéniques, qui ont pu influencer directement ou indirectement l'auteur dramatique " (Pavis 1996 : 334).

4. En sont exclues Cardenio et Love's Labours Won, dont les textes n'ont jamais été retrouvés et The Two Noble Kinsmen, pièce écrite en collaboration avec John Fletcher, ainsi que tous les textes que la recherche désigne aujourd'hui sous le nom d' " apocryphes ", c'est-à-dire ceux pour lesquels William Shakespeare est considéré comme un collaborateur mineur.

5. Contre 105 d'origine anglaise (anglais ou latin), 21 d'origine classique (latin ou grec), 19 de langue italienne, 10 de langue française et 5 de langue allemande. Ne sont pas comptabilisés ici les documents épistolaires.

6. Les autres œuvres sont la Comedia Eufemia de Lope de Rueda, The Mirror of Knighthood, traduction de Espejo de Príncipes y Cavalleros et Amadis de Gaule.

7. L'intertextualité est la « relation de coprésence entre deux ou plusieurs textes, eidétiquement et le plus souvent, par la présence effective d'un texte dans un autre » (Genette $1982: 8$ ).

8. Selon Gérard Genette, «la transtextualité, ou transcendance textuelle du texte ", c'est-à-dire «tout ce qui le met en relation, manifeste ou secrète, avec d'autres textes " est l'objet de la poétique (Genette 1982 : 7). Il existe cinq types de relations transtextuelles : l'intertextualité, la paratextualité, la métatextualité, l'architextualité et l'hypertextualité.

9. Je tiens à remercier ici Sabine Schuelting, éditrice du Shakespeare Jahrbuch, qui m'a permis d'avoir accès à certains de ces articles. Voir http://shakespeare-gesellschaft.de/en/ jahrbuch.html.

10. « $[\mathrm{T}]$ he extent of an author's acquaintance with the language of a foreign country is obviously an important factor in considering the possible influences exercised upon him by that country's literature."

11. "Shakespeare's knowledge of Spain seems to have been that of the intelligent London citizen. » 
12. "The Spanish words and expressions in the plays of Shakespeare can be counted upon the fingers of one hand, and they are either corruptions, or, (in two instances) caricatures of Spanish celebrities. "

13. La coprésence peut revêtir plusieurs formes effectives selon la nature du lien plus ou moins explicite ou plus ou moins littéral : il peut s'agir de la citation, du plagiat ou de l'allusion (Genette $1982: 8)$.

14. Dans l'ordre de citation, la Comedia Eufemia et la Comedia de los Engañados de Lope de Rueda, Castelvines y Monteses de Lope de Vega, la Comedia de Rubena de Gil Vincente, la Celestina, le Conde Lucanor de Don Juan Manuel, la Segunda Celestina de Feliciano de Silva, la Historia de Grisel y Mirabella de Juan de Flores, le Lazarillo de Tormes, l'Examen de Ingenios de Juan Huarte, le Don Quixote de Miguel de Cervantes, la Diana de Jorge de Montemayor, la série des Amadis de Feliciano de Silva et l'Espejo de Principes y Cavalleros.

15. Il n'est pas exclu de déceler en germe ici les théories qui seront développées un siècle plus tard par Walter Cohen dans Drama of a Nation.

16. "[...] the same characters, plots, and places have been utilized by these men in the construction of some of their dramatic productions, and, being contemporaries, an impression may be created that a considerable amount of borrowing-call it plagiarism if you wish-must have been indulged in. [...] [A]s Shakespeare has suffered more or less from the imputation of non-originality, it may be important to consider to what extent, if any, he was indebted to Lope de Vega and his school.»

17. Sept chez Frey et vingt-huit chez Thomas.

18. "Would he then find it necessary to copy from such unfaithful forms? Would he derive any benefit by imitating such unrealistic prototypes? Most assuredly not! »

19. «[...] aparte de que no abarca más que algunos de los aspectos, es demasiado compendioso y superficial; $\mathrm{y}$, al concluir su lectura, deja la impresión de ser un tanto dogmatico y conservador. » 20. «[...] the opinions of [...] Frey have become dominant and deterred research. »

21. «[...] the unities of time and place were not observed, and historical facts were perverted for the purpose of adaptation. [...] But these anachronisms and blunders are of rare occurrence in Shakespeare's works. »

22. "Censored Protestant authors might hesitate to cite suspected Spanish authorities.»

23. "[Shakespeare] had ample sources of inspiration for Romeo and Juliet in his English predecessors in the same field, Brooke and Painter. »

24. «Cualquier toma de contacto con personajes hispanos o hispanizantes, o con la proyección de estos en sus obras, ideas y manifestaciones de cualquier índole, todo empleo y alusión que haya hecho, toda actitud que haya tomado, y toda mención - expresa o tácita - que haga referencia a España, a los españoles, a lo español y al castellano, serán aquí tomados en cuenta. Las influencias directas o indirectas son solamente un modo más de estar presente, pero no el único » (Duque $1991: 16)$.

25. «[...] the ethos of Spanish cruelty in Inquisition and conquest became coupled with Spanish "darkness", the ethnos of its "most bastardly" origins as a nation composed of no one single race but as admixture of Roman, African, Moor and Jew. This confluence of discourses produces the "ethnopoesis" of Spain's "covetous, amoral, and unworkable" imperialism, in contrast to England's idealized "less internationally ambitious and (presumably) less corruptible sovereign state"."

26. «Hispanists and Anglicists working in both areas of early modern studies look forward to new offerings in the relatively under-explored field of comparative Anglo-Hispanic literature. » 27. "While isolated work has emerged since the 1980s [...] more work is needed to properly situate, historicize, and recognize the dialogic potential of both literatures together. » 


\section{RÉSUMÉS}

L'objet d'étude de cet article est l'évolution historiographique des outils théoriques et des pratiques d'analyse textuelle utilisés dans l'étude des hypotextes shakespeariens, traditionnellement appelés "sources». Le corpus est constitué de quelques exemples d'origine espagnole dans le théâtre de William Shakespeare tirés des travaux des théoriciens des sources shakespeariennes (Henry Thomas, Geoffrey Bullough ou encore Kenneth Muir). L'objectif est de démontrer que l'élargissement des possibles et le renouveau des perspectives d'analyse résident dans le glissement de la notion de « source » vers la notion d'« hypotexte » qui permet d'aborder la question sous l'angle de nouvelles méthodologies comme, entre autres, la transtextualité ou la théorie de l'adaptation.

This article deals with the historiographical examination of the evolution of theoretical tools and textual analysis practices used in the study of Shakespearean hypotexts, traditionally called "sources". The corpus is made up of a few examples of Spanish origin in the plays of William Shakespeare taken from the works of critics of Shakespearean sources (Henry Thomas, Geoffrey Bullough or Kenneth Muir...) This paper aims at establishing the shift from the notion of "source" to the notion of "hypotext" as widening possibilities and renewing analysis perspectives, the notion of "hypotext" enabling research to tackle this matter with new methodologies, such as, among others, transtextuality and adaptation theories.

\section{INDEX}

Mots-clés : textes espagnols, historiographie, transtextualité, Shakespeare (William), adaptation Keywords : Spanish texts, historiography, transtextuality, adaptation, Shakespeare (William)

\section{AUTEUR}

\section{ANNA DEMOUX}

Doctorante, Université Blaise Pascal Clermont-Ferrand 2, CERHAC (UMR 5037) 